\title{
Surgery after transcatheter aortic valve interventions
}

\author{
Christopher R. Burke, MD, Shakirat O. Oyetunji, MD, MPH, and Gabriel S. Aldea, MD
}

Associate Editor's Introduction-Aortic valve replacement, whether performed open or percutaneously, inherently trades the native valve disease for a new disease by virtue of the prosthetic valve. Both approaches have pitfalls and complications early, from the procedure itself (eg, heart block, perivalvular leak, ventricular injury, coronary artery ostial impingement), and later (eg, prosthetic valve endocarditis, structural valve deterioration [SVD]). With transcatheter aortic valve replacement (TAVR), even after successful deployment, the biological nature of the valve implies that SVD will eventually occur, and the risks of thrombosis and prosthetic valve endocarditis remain. With the progression of TAVR use in lower-risk cohorts, including younger patients, the long-term consequences should remain in the back of the surgeon's mind. In this invited review, the authors have provided the readership with a concise summary of issues facing surgeons involved in TAVR in both the acute and long-term periods. This summary is just that, a summary of the potential issues and some potential solutions, but it reminds us that no procedure or technique is free of complications. Knowledge and understanding of these potential complications are critical in the optimal care of our patients.

\section{Abe DeAnda Jr, MD}

The use of transcatheter aortic valve replacement (TAVR) has increased significantly in the past 5 years. According to the TVT registry, TAVR surpassed 70,000 implants in calendar year 2019 in the United States. Compared with isolated surgical aortic valve replacement (SAVR), TAVR is now implanted at a $>2: 1$ ratio and, because of prospective randomized trials, ${ }^{1-3}$ in an increasingly younger and lower-risk patient population. To date, with limited longterm follow-up, freedom from structural heart deterioration has been acceptable in intermediate- and high-risk populations. However, given that all biological valves are expected to fail with sufficiently long follow-up times, we anticipate that cardiac surgeons will need to acquire a better understanding of both acute and chronic complications related

From the Division of Cardiothoracic Surgery, Department of Surgery, University of Washington, Seattle, Wash.

Received for publication Dec 1, 2020; accepted for publication Dec 1, 2020; available ahead of print Jan 6, 2021

Address for reprints: Gabriel S. Aldea, MD, Cardiothoracic Surgery, University of Washington Med School, Box 356310, 1959 NE Pacific St, Seattle, WA 981956310 (E-mail: aldea@u.washington.edu or aldea@uw.edu).

JTCVS Techniques 2021;6:54-8

2666-2507

Copyright $@ 2021$ The Authors. Published by Elsevier Inc. on behalf of The American Association for Thoracic Surgery. This is an open access article under the CC BY-NCND license (http://creativecommons.org/licenses/by-nc-nd/4.0/).

https://doi.org/10.1016/j.xjtc.2020.12.038

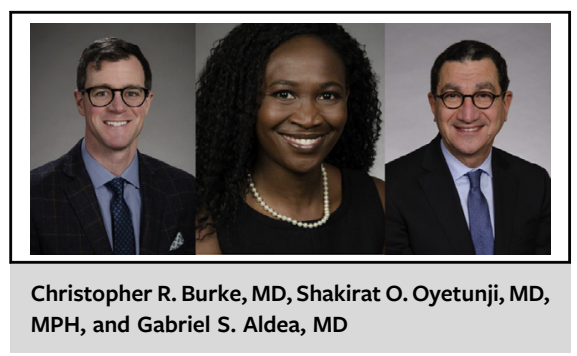

CENTRAL MESSAGE

Cardiac surgeons will need to gain expertise with a broad, complex spectrum of clinical scenarios and techniques to address open surgical interventions after transcatheter valve Implantations.

See Commentaries on pages 59 and 61.

to TAVR, as well as an expanded skill set to successfully treat these complications. Familiarity with the characteristics of the different transcatheter heart valve (THV) prostheses and careful serial echocardiographic and clinical surveillance and follow-up are critical. Although these events are currently rare, given the high implantation rates, a significant increase in surgical reintervention is anticipated over the next decade.

\section{ACUTE INTERVENTION}

The incidence of acute (so-called "emergency surgical bailout") open intervention after TAVR remains relatively low $(1 \%-3 \%)$, but this intervention is associated with significantly increased morbidity and mortality. ${ }^{4-6}$ Acute and rapid diagnosis and preparedness and experience of the team to address these rare but complex clinical scenarios are paramount. Data from the TVT registry identified the most common reasons for acute surgical bailout following TAVR as device migration (22.3\%), cardiac perforation (with pericardial effusion and tamponade; $19.4 \%$ ), and annular/root rupture (14.2\%). Other causes include type A dissection and coronary occlusion.

Although the need for emergency "bailout" surgical intervention following TAVR is rare, the candidacy and prognosis for such salvage interventions, especially after catastrophic complications, should be formally discussed by the multidisciplinary team with the patient and family 
before any planned TAVR procedure. Candidacy for such salvage interventions is also formally and specifically reviewed as part of the Surgical Care and Outcomes Assessment Program preprocedure briefing by the entire procedural team.

\section{Device Migration}

The incidence of this complication has decreased significantly with the evolution of enhanced preoperative imaging, more precise valve sizing, and repositionable transcatheter valves. These are more common in the setting of self-expanding valves, aortic insufficiency, large annuli, minimal annular calcification, and bicuspid valve pathology. ${ }^{8}$ Dislodged valves often can be retrieved percutaneously from the ascending aorta with deployment of the valve in the descending thoracic aorta distal to the left subclavian artery, but left ventricular dislodgment invariably requires an open retrieval and cardiopulmonary bypass.

\section{Cardiac Perforation}

Although cardiac perforations are more common in the right heart (with transvenous pacemaker manipulations), mortality is higher with left-sided cardiac perforation. ${ }^{9}$ Most cardiac chamber wire perforations respond to percutaneous pericardial drainage. A small subset of patients require open operative intervention and direct repair. Persistent bleeding following pericardial catheter drainage and reversal of anticoagulation should prompt consideration for urgent open repair. Surgical repair often can be accomplished with a direct suture repair and rarely requires the use of cardiopulmonary bypass.

\section{Annular Rupture}

Annular rupture represents a truly devastating complication following TAVR. Risk factors for annular rupture include a heavily calcified (asymmetric) aortic root/ annulus, left ventricular outflow tract (LVOT) calcification, oversizing or overinflation of THV, and use of a balloonexpandable rather than self-expanding TAVR valve. ${ }^{10}$ Rupture can occur anywhere within the "TAVR landing zone" and can be subannular, annular, at the level of the aortic root (sinus and sinotubular junction), or a combination of these. Clinical presentation is commonly that of hemodynamic instability and a new pericardial effusion. Stabilizing the patient requires a rapid and accurate diagnosis. The diagnosis is frequently evident on root aortography, but transesophageal echocardiography and, in more subacute circumstances, computed tomography angiography, can be helpful (Figure 1,A). Rupture in the various areas of the landing zone can lead to varied clinical presentations. Rupture within the LVOT can cause injury to the ventricular septum or free wall, occasionally leading to acute ventricular septal defect. Annular rupture is sometimes contained and in some cases can be managed expectantly. Rupture of the aortic root can cause catastrophic bleeding or focal dissection within the root (Figure 1, B). Disruption or partial detachment of the coronary arteries from the free wall and rupture of the aorta can occur.

Pericardial drain placement is a critical initial step before planning and providing definitive therapy in patients with no contraindications who were identified as candidates for "salvage" intervention before the TAVR procedure. Although sternotomy and central cannulation are preferable whenever possible, some patients require rapid recognition and initiation of peripheral cardiopulmonary bypass support to stabilize, although definitive repair is often a salvage attempt. Arterial cannulation frequently can be expedited by inserting the arterial inflow cannula into the previously placed TAVR femoral sheath (in $16 \mathrm{~F}$ or larger sheaths). Treatment invariably mandates LVOT or root reconstruction and an aortic root replacement (our preference is a BioBentall with a freestyle root or homograft). Coronary artery
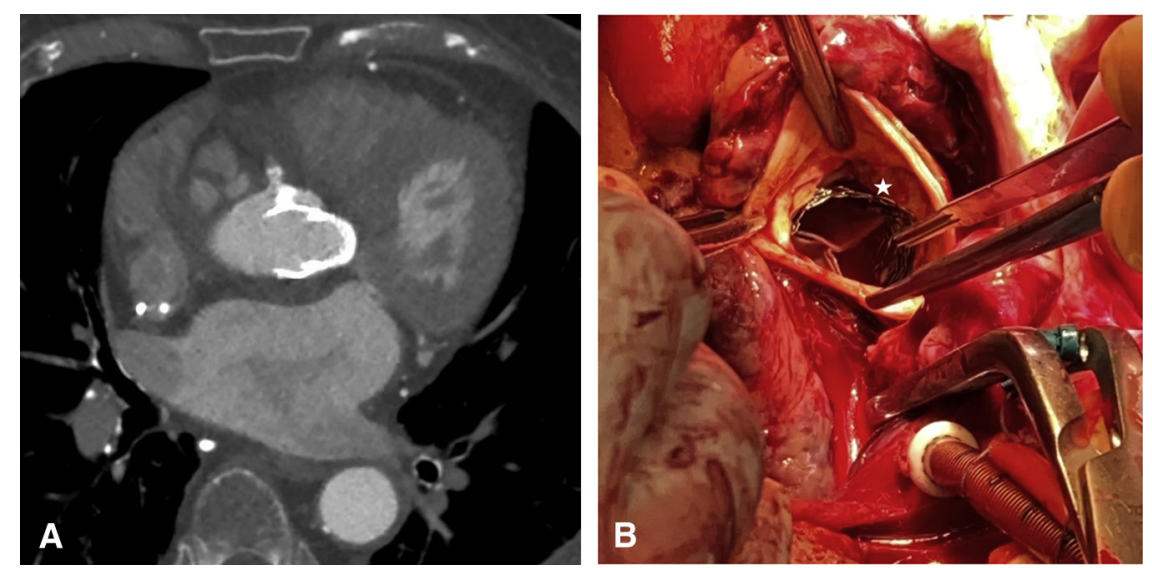

FIGURE 1. A, Computed tomography angiography after transcatheter aortic valve replacement (TAVR) showing rupture and a pseudoaneurysm cavity near the ostium of the right coronary artery. B, Intraoperative image showing complete aortic root disruption into the right coronary sinus (star) following TAVR. 
ostia can be calcified but also significantly displaced or even injured/avulsed by the rapidly expanding hematoma, and coronary bypass must be considered when primary reimplantation of coronary buttons is not feasible. Aortic hematoma is typically limited to the root, and central aortic cannulation in the distal ascending aorta is feasible. The explant of the TAVR prosthesis is technically fairly straightforward in the acute setting (very recent implantation and no adhesions/incorporation), but complex root replacement is almost always required, with principles similar to those in repair of type A dissection involving the aortic root. Mortality is roughly $50 \%$, related to patient hemodynamic instability, underlying cardiac and general comorbidities, and potential difficulties with and the extent of repair. ${ }^{5,10}$

\section{CHRONIC INTERVENTIONS \\ Paravalvular Leak}

Although many paravalvular leaks (PVLs) are amenable to percutaneous therapy using a variety of plugs, these less invasive techniques can be associated with incomplete resolution of PVL, as well as with a persistent or increased risk of hemolysis. Persistent or large PVLs typically require open surgical approaches with explantation of a transcatheter valve prosthesis and redo surgical AVR. ${ }^{11}$ In a single-center experience at the University of
Michigan over a 9-year period, the leading indication for valve explantation was severe symptomatic PVL, occurring in $41 \%$ of their THV explants. In their series, the median time from the index TAVR to THV explantation was 96 days (interquartile range, $69-438$ days). ${ }^{11}$

Again, the incidence of PVL has decreased significantly in the past several years with the introduction and wide adoption of third-generation transcatheter aortic valves (annular skirts). ${ }^{1,2}$

\section{Prosthetic Valve Bacterial Endocarditis}

The incidence of prosthetic valve endocarditis (PVE) following TAVR is similar to that of SAVR. ${ }^{12,13}$ There is a small peak in the first 6 months, but after that initial perioperative period, the incidence is approximately $5 \%$ in the first 5 years with a peak ( $65 \%$ of all infections) noted in the first year and is associated with a risk-adjusted 3-fold increase in mortality. ${ }^{12,13}$ Although staphylococcal organisms predominate, enterococcal organisms are also common. Clinical presentations are similar to those of surgical PVE, vary somewhat between self-expanding and balloon-expanded transcatheter valves, but the presence of prosthetic annular and subannular material increases risk of annular and aortic-mitral curtail involvement and abscess formation (Figure 2, A-D). Surgical explantation rates of
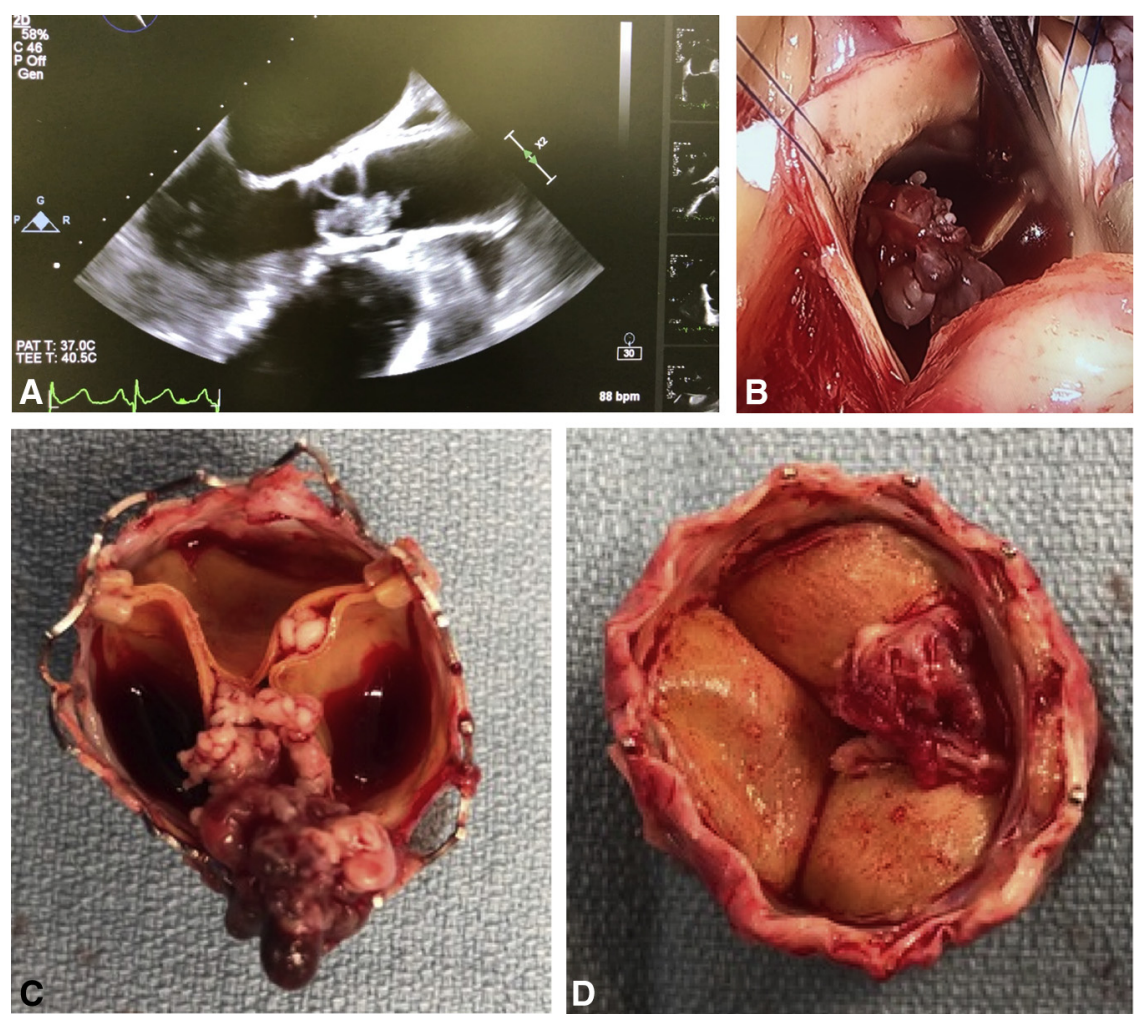

FIGURE 2. A, Transesophageal echocardiogram showing large mobile endocarditic vegetation on a Sapien S3 transcatheter aortic valve replacement (TAVR) prosthesis. B, Intraoperative explantation of a Sapien S3 (TAVR) valve. C, Explanted TAVR Sapien S3 valve with Candida endocarditis and vegetation, aortic perspective. D, Explanted TAVR Sapien S3 valve with Candida endocarditis and vegetation, ventricular perspective. 
transcatheter PVE remain low, performed in $<15 \%$ of patients and may reflect a significant selection bias because of patients' high/prohibitive comorbidities. Mortality of both medical therapy and SAVR in this high-risk patient cohort remains high and varies from $36 \%$ to $>60 \%$. TAVR PVE was increased in younger patients, males, and in persons with diabetes, residual aortic insufficiency, heart failure, and chronic renal disease. ${ }^{14-16}$

\section{Prosthetic SVD and Hemodynamic Valve Degeneration}

The incidence of leaflet motion abnormalities and the potential for subannular thrombus and pannus formation appear to be higher for TAVR valves and once diagnosed, may be ameliorated by the use of oral anticoagulation rather than dual antiplatelet therapies. ${ }^{17,18}$ As with SAVR prostheses, close clinical and echocardiographic follow-up of the valve is essential. Prosthetic leaflet thickening, decreased leaflet mobility, and increasing transvalvular gradients should prompt repeat TEE and multidetector computed tomography imaging. The clinical incidence of SVD and hemodynamic valve degeneration are currently underestimated. ${ }^{19,20}$ Smaller TAVR prostheses, patient-prosthesis mismatch, and valve-invalve TAVR may be more prone to this complication. Larger TAVR prostheses may accommodate a repeat TAVR valve-in-valve, but surgical explantation of smaller THV prostheses should be considered along with surgical adjuncts to deal with a small LVOT and aortic root more definitively.

TAVR prosthesis explantation is more technically challenging, owing to the adherence of native leaflets to the TAVR prosthesis and even more so to endothelial ingrowth of the THV valve into the annulus, subannular mechanism (mitral valve and membranous septum of the LVOT), and aorta (especially with supra-annular valves, such as the CoreValve). Although an endarterectomy of the prosthetic valve cage is possible, root (Bentall) replacement and with smaller anatomic annuli, root enlargement, is often required. Aortotomy is typically performed at or just above the top level of the prosthetic cage, and for CoreValve Evolut $\mathrm{R}$ and PRO require an extensive, frequently tedious, and meticulous endarterectomy dissection of the THV from the native aorta, from the displaced native or prosthetic aortic valve leaflets, annulus, and mitral valve. Prosthetic valve deterioration requiring surgical explantation is expected to be more common as THV prostheses implanted in younger patients as salvage temporizing therapies or as definitive treatment (Figure 3, $A$ and $B$ ).

\section{Late Device Embolization}

Similar to acute device migration, the incidence of this complication has decreased significantly with enhanced preoperative imaging, more precise valve sizing, and use of repositionable THVs. In a systematic review of 70 publications evaluating the mechanisms and management of TAVR failure, Mylotte and colleagues ${ }^{21}$ reported late valve embolization in $21 \%$ of their cases. Risk factors associated with late device embolization are similar to those seen in acute device migration, including low prosthesis implantation, large annulus, valve undersizing, bicuspid valve pathology, aortic insufficiency, and minimal annulus calcification. Valve embolization mandated surgical intervention in $80 \%$ of patients.

Device migration can manifest with associated abnormalities, such as severe mitral regurgitation, tricuspid regurgitation, and ventricular septal defect.

\section{Outcomes}

Outcomes of transcatheter valve explantation are related to urgency, patient pathoanatomic circumstances, presence of comorbidities, and the experience of the surgical and perioperative teams. ${ }^{5-8,11}$
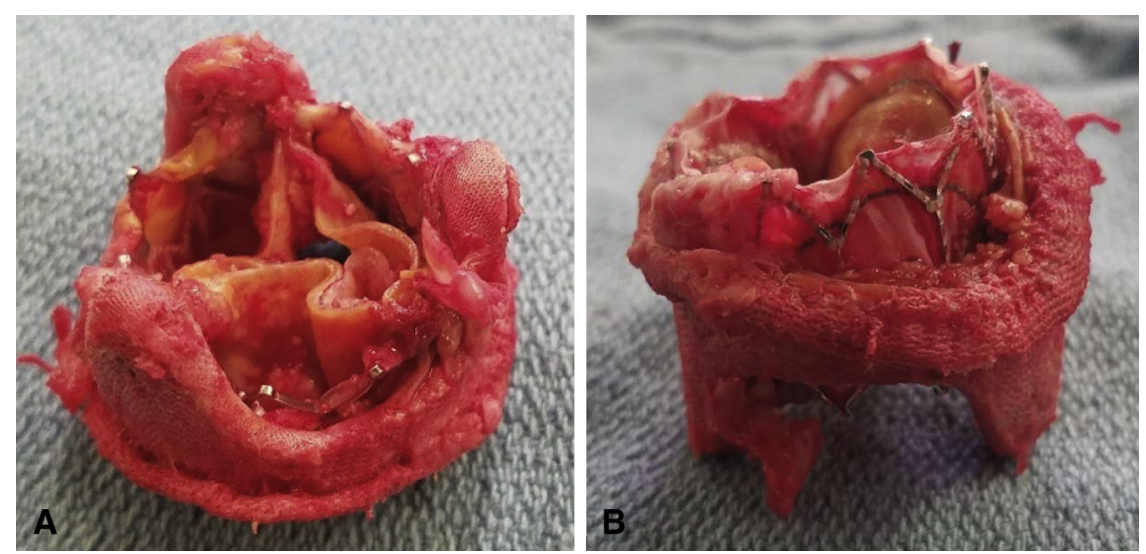

FIGURE 3. A, Prosthetic transcatheter aortic valve replacement (TAVR) valve degeneration (Sapien XT in prior Mosaic valve), aortic perspective. B, Prosthetic TAVR valve degeneration (Sapien XT in prior Mosaic valve), ventricular perspective. 


\section{CONCLUSIONS}

Cardiac surgeons will need to gain familiarity with the specific characteristics of the growing spectrum, specific characteristics, and evolution of transcatheter valve devices as the incidence of prosthetic valve dysfunction increases with expanded use over time. Explantation techniques are tailored to the implanted transcatheter device and to the patient's individual pathology and anatomy. Expertise in aortic endarterectomy, advanced aortic root enlargement and replacement, and coronary artery revascularization is needed to manage a population of patients with potentially complex comorbidities. Outcomes to date are acceptable and should improve with growing experience and expertise.

\section{Conflict of Interest Statement}

The authors reported no conflicts of interest.

The Journal policy requires editors and reviewers to disclose conflicts of interest and to decline handling or reviewing manuscripts for which they may have a conflict of interest. The editors and reviewers of this article have no conflicts of interest.

\section{References}

1. Mack MJ, Leon MB, Thourani VH, Makkar R, Kodali SK, Russo M, et al. Transcatheter aortic-valve replacement with a balloon-expandable valve in low-risk patients. N Engl J Med. 2019;380:1695-705.

2. Popma JJ, Deeb GM, Yakubov SJ, Mumtaz M, Gada H, O'Hair D, et al. Transcatheter aortic-valve replacement with a self-expanding valve in low-risk patients. N Engl J Med. 2019;380:1706-15.

3. Kolte D, Vlahakes GJ, Palacios IF, Sakhuja R, Passeri JJ, Inglessis I, et al. Transcatheter versus surgical aortic valve replacement in low-risk patients. J Am Coll Cardiol. 2019;74:1532-40.

4. Hein R, Abdel-Wahab M, Sievert H, Kuck KH, Voehringer M, Hambrecht R, et al. Outcome of patients after emergency conversion from transcatheter aortic valve implantation to surgery. EuroIntervention. 2013;9:446-51.

5. Walther T, Hamm CW, Schuler G, Berkowitsch A, Kötting J, Mangner N, et al. Perioperative results and complications in 15,964 transcatheter aortic valve replacements: prospective data from the GARY registry. J Am Coll Cardiol. 2015;65:2173-80.

6. Trenkwalder T, Pellegrini C, Holzamer A, Philipp A, Rheude T, Michel J, et al. Emergency extracorporeal membrane oxygenation in transcatheter aortic valve implantation: a two-center experience of incidence, outcome and temporal trends from 2010 to 2015. J Am Coll Cardiol. 2019;74:1532-40.
7. Pineda AM, Harrison JK, Kleiman NS, Rihal CS, Kodali SK, Kirtane AJ, et al. Incidence and outcomes of surgical bailout during TAVR: insights from the STS/ACC TVT registry. JACC Cardiovasc Interv. 2019;12:1751-64.

8. Guerrero M, Urena M, Himbert D, Wang DD, Eleid M, Kodali S, et al. 1-year outcomes of transcatheter mitral valve replacement in patients with severe mitral annular calcification. J Am Coll Cardiol. 2018;71:1841-53.

9. Provaznik Z, Holzamer A, Camboni D, Rupprecht L, Resch M, Wittmann S, et al. Perforation of myocardial wall and great vessels after cardiovascular interventions: a 5-year analysis. J Thorac Dis. 2017;9:5288-94.

10. Pasic M, Unbehaun A, Buz S, Drews T, Hetzer R. Annular rupture during transcatheter aortic valve replacement: classification, pathophysiology, diagnostics, treatment approaches, and prevention. JACC Cardiovasc Interv. 2015;8(1 Pt A):1-9.

11. Fukuhara S, Brescia AA, Shiomi S, Rosati CM, Yang B, Kim KM, et al. Surgical explantation of transcatheter aortic bioprostheses: results and clinical implications. J Thorac Cardiovasc Surg. January 12, 2020 [Epub ahead of print].

12. Mentias A, Girotra S, Desai MY, Horwitz PA, Rossen JD, Saad M, et al. Incidence, predictors, and outcomes of endocarditis after transcatheter aortic valve replacement in the United States. JACC Cardiovasc Interv. 2020;13: 1973-82.

13. Summers MR, Leon MB, Smith CR, Kodali SK, Thourani VH, Herrmann HC, et al. Prosthetic valve endocarditis after TAVR and SAVR: insights from the PARTNER trials. Circulation. 2019;140:1984-94.

14. Kuttamperoor F, Yandrapalli S, Siddhamsetti S, Frishman WH, Tang GHL. Infectious endocarditis after transcatheter aortic valve replacement: epidemiology and outcomes. Cardiol Rev. 2019;27:236-41.

15. Latib A, Naim C, De Bonis M, Sinning JM, Maisano F, Barbanti M, et al. TAVRassociated prosthetic valve infective endocarditis: results of a large, multicenter registry. J Am Coll Cardiol. 2014;64:2176-8.

16. Regueiro A, Linke A, Latib A, Ihlemann N, Urena M, Walther T, et al. Association between transcatheter aortic valve replacement and subsequent bacterial endocarditis and in-hospital death. JAMA. 2016;316:1083-92.

17. Chakravarty T, Søndergaard L, Friedman J, De Backer O, Berman D, Kofoed KF, et al. Subclinical leaflet thrombosis in surgical and transcatheter bioprosthetic aortic valves: an observational study. Lancet. 2017;389:2383-92.

18. Rosseel L, De Backer O, Søndergaard L. Clinical valve thrombosis and subclinical leaflet thrombosis following transcatheter aortic valve replacement: is there a need for a patient-tailored antithrombotic therapy? Front Cardiovasc Med. 2019; $6: 44$.

19. Dvir D, Bourguignon T, Otto CM, Hahn RT, Rosenhek R, Webb JG, et al. Standardized definition of structural valve degeneration for surgical and transcatheter bioprosthetic aortic valves. Circulation. 2018;137:388-99.

20. Smith D. Bioprosthetic valve durability: TAVR versus SAVR using different definitions of valve deterioration. Int J Cardiol. 2018;268:176-8.

21. Mylotte D, Andalib A, Thériault-Lauzier P, Dorfmeister M, Girgis M, Alharbi W, et al. Transcatheter heart valve failure: a systematic review. Eur Heart J. 2015;36: 1306-27.

Key Words: cardiac surgery, prior TAVR 